2012

\title{
Complete Multipartite Graphs and the Relaxed Coloring Game
}

Charles Dunn

Linfield College

Follow this and additional works at: https://digitalcommons.linfield.edu/mathfac_pubs

Part of the Discrete Mathematics and Combinatorics Commons

\section{DigitalCommons@Linfield Citation}

Dunn, Charles, "Complete Multipartite Graphs and the Relaxed Coloring Game" (2012). Faculty

Publications. Accepted Version. Submission 5.

https://digitalcommons.linfield.edu/mathfac_pubs/5

This Accepted Version is protected by copyright and/or related rights. It is brought to you for free via open access, courtesy of DigitalCommons@Linfield, with permission from the rights-holder(s). Your use of this Accepted Version must comply with the Terms of Use for material posted in DigitalCommons@Linfield, or with other stated terms (such as a Creative Commons license) indicated in the record and/or on the work itself. For more information, or if you have questions about permitted uses, please contact digitalcommons@linfield.edu. 


\title{
COMPLETE MULTIPARTITE GRAPHS AND THE RELAXED COLORING GAME
}

\author{
CHARLES DUNN
}

\begin{abstract}
Let $k$ be a positive integer, $d$ be a nonnegative integer, and $G$ be a finite graph. Two players, Alice and Bob, play a game on $G$ by coloring the uncolored vertices with colors from a set $X$ of $k$ colors. At all times, the subgraph induced by a color class must have maximum degree at most $d$. Alice wins the game if all vertices are eventually colored; otherwise, Bob wins. The least $k$ such that Alice has a winning strategy is called the $d$-relaxed game chromatic number of $G$, denoted $\chi_{\mathrm{g}}^{d}(G)$. It is known that there exist graphs such that $\chi_{\mathrm{g}}^{0}(G)=3$, but $\chi_{\mathrm{g}}^{1}(G)>3$. We will show that for all positive integers $m$, there exists a complete multipartite graph $G$ such that $m \leq \chi_{\mathrm{g}}^{0}(G)<\chi_{\mathrm{g}}^{1}(G)$.
\end{abstract}

\section{IntRODUCTION}

The $(k, d)$-relaxed coloring game is played on a finite graph $G$ with a finite set of $k$ colors $X$, where $d$ is a nonnegative integer. Two players, Alice and Bob, alternate coloring the uncolored vertices of $G$, with Alice going first. At any point in the game for any $\alpha \in X$, let $C_{\alpha}$ be the set of vertices colored $\alpha$ at that point. We say that a color $\alpha \in X$ is legal for the uncolored vertex $u$, if after $u$ is colored with $\alpha$ we have that $\Delta\left(G\left[C_{\alpha}\right]\right) \leq d$; that is, the subgraph induced by all vertices colored $\alpha$ has maximum degree at most $d$. On each turn, players must color vertices with legal colors. Alice wins the game if all vertices are colored; otherwise, Bob wins when there is an uncolored vertex for which no legal color exists. The least $k$ for which Alice has a winning strategy for this game on $G$ is called the d-relaxed game chromatic number of $G$, denoted $\chi_{\mathrm{g}}^{d}(G)$. When $d=0$, we refer simply to the game chromatic number of $G$ and write $\chi_{\mathrm{g}}(G)$. The game chromatic number was first introduced by Bodlaender in [2]. It has since been studied extensively,

Date: May 2010.

2000 Mathematics Subject Classification. 05.

Key words and phrases. competitive coloring, relaxed coloring, multipartite graphs. 
including in $[4,8,10]$. The relaxed game chromatic number has been considered in $[3,5,6,7,9]$.

Suppose that Alice and Bob are playing the $(k, d)$-relaxed coloring game on a graph $G$. For the purposes of analyzing strategies for Alice or Bob, we note that a color $\alpha$ is legal for an uncolored vertex $u$ if the following two conditions hold:

(1) The vertex $u$ has at most $d$ neighbors already colored $\alpha$.

(2) If $v$ is a neighbor of $u$ and $v$ is already colored $\alpha$, then $v$ has at most $d-1$ neighbors already colored $\alpha$.

It has been well established that the parameter $\chi_{\mathrm{g}}(G)$ has some interesting and possibly unexpected properties. For example, while it is true that if $H$ is a subgraph of $G$, then $\chi(H) \leq \chi(G)$, it is not necessarily the case that $\chi_{\mathrm{g}}(H) \leq \chi_{\mathrm{g}}(G)$. For example, as discussed in [1], if $G=K_{n, n}$ and $n \geq 2$ we have that $\chi_{\mathrm{g}}(G)=3$. However, if $M$ is any perfect matching in $G$, then $\chi_{\mathrm{g}}(G-M)=n$. So for $n \geq 4$, $\chi_{\mathrm{g}}(G-M)>\chi_{\mathrm{g}}(G)$. In addition, with related reasoning, it is known that if $G=K_{n, n}$ with $n \geq 2$, then $\chi_{\mathrm{g}}^{1}(G)=n$. So for $n \geq 4$, there is a class of graphs for which $\chi_{\mathrm{g}}^{0}(G)=3$ but $\chi_{\mathrm{g}}^{1}(G) \geq 4$. In light of this latter example, we consider the following question:

Question 1. For every $m \in \mathbb{N}$, does there exist a graph $G$ such that $m \leq \chi_{\mathrm{g}}(G)<\chi_{\mathrm{g}}^{1}(G)$ ?

Generalizing from bipartite graphs to complete multipartite graphs settles Question 1 in the affirmative.

For ease of notation, we denote the complete $r$-partite graph with each partite set of size $n$ by $K_{r * n}$. We will also label the partite sets in the order that they receive their first colored vertex. In this manner, for $j \in\{1,2, \ldots, r\}$ we denote the $j$-th partite set of $K_{r * n}$ to receive its first colored vertex by $P_{j}$.

\section{The Game Chromatic Number}

We begin by showing that the game chromatic number of $K_{r * n}$ is always bounded above by a function of $r$. We will also show that the upper bound is tight. We will show this with two lemmas.

Lemma 1. Let $r$ and $n$ be positive integers, and let $G=K_{r * n}$. Then $\chi_{\mathrm{g}}(G) \leq 2 r-1$. Moreover, if $n=3$ and $r \geq 3$, then $\chi_{\mathrm{g}}(G) \leq 2 r-2$.

Proof. Suppose Alice and Bob are playing the $(2 r-1,0)$-coloring game on $G$. We will show that Alice has a winning strategy for this game. We begin by making a simple observation. Suppose that at some point in the game vertex $v \in P_{i}$ is colored with color $\alpha \in X$. Now, for the 
remainder of the game, the color $\alpha$ is legal for any uncolored vertex in $P_{i}$ and not legal for any uncolored vertex outside $P_{i}$. Thus, once each partite set has a colored vertex, every vertex will have a legal color and Alice will eventually win.

On each of her turns, Alice will color a vertex in a new partite set. By her $r$-th turn, Alice will have ensured that each partite set has a colored vertex. At this time, she will have used $r$ colors and Bob could have used at most $r-1$ other colors. Thus Alice will win.

Now suppose $n=3, r \geq 3$, and that Alice and Bob are playing the $(2 r-2,0)$-coloring game on $G$. Assume that $X=\{1,2, \ldots, 2 r-2\}$. On her first turn Alice will color a vertex with 1. On her second turn, she will color in a partite set that already has a colored vertex, either in the partite set in which she colored on her first turn, or the one in which Bob has just colored. She will repeat a color that has already been used in that partite set. Hence, using the fact that $n=3$, by the end of Bob's second turn, at least two partite sets have colored vertices and at most 3 colors have been used. On her next $r-2$ turns, Alice will ensure that each of the remaining partite sets has a colored vertex. So the number of colors that Bob can use during this portion of the game is $r-3$. Thus the number of colors used will be at most $3+(r-2)+(r-3)=2 r-2$. Since each partite set has a colored vertex by this point, Alice will win the game.

Next we show that the above upper bounds are in fact lower bounds as well.

Lemma 2. Let $r$ and $n$ be positive integers, and let $G=K_{r * n}$. If $n \geq 2$ and $n \neq 3$, then $\chi_{\mathrm{g}}(G) \geq 2 r-1$. If $n=3$ and $r \geq 3$, then $\chi_{\mathrm{g}}(G) \geq 2 r-2$.

Proof. Suppose that Alice and Bob are playing the $(k, 0)$-coloring game on $G$ where $k \leq 2 r-1$. Recall that we will assume that the partite sets are numbered according to the order in which they receive their first colored vertex. Bob's strategy will be as follows. Suppose that Alice has just colored a vertex in $P_{i}$. If there exists an uncolored vertex in $P_{i}$, Bob will color such a vertex with a new color. If $P_{i}$ has no remaining uncolored vertices, Bob will color in a partite set with a colored vertex, if possible, preferably using a new color. Otherwise, Bob will color in a partite set with no colored vertices.

First suppose that Bob is never forced to be the first to color in a partite set. Then all but the last partite set will have at least two different colors used in them. Since no color can be used in more than one partite set, the number of colors necessary for Alice to win is 
$2(r-1)+1=2 r-1$. Note that if $n$ is even, this will always be the case, as Bob can always follow Alice, coloring in the same partite as she did on her most recent turn. So if $n$ is even and $k \leq 2 r-2$, then Bob will win.

We may now assume that $n$ is odd, $n \geq 3$ and that at some time in the game, Bob is forced to be the first to color in a partite set. Consider the last time in the game that this happens. Suppose Bob is forced to color first in $P_{i+1}$. Since Bob's strategy has him preferring to color in partite sets with colored vertices, we have that all $n i$ vertices in $\cup_{j=1}^{i} P_{j}$ are already colored. Note that the colors of the first vertices colored in each of these partite sets are distinct and that Bob has colored at most half of them. Thus, in addition, the number of colors that Bob has used on the remaining vertices in $\cup_{j=1}^{i} P_{j}$ is at least $\frac{(n-1) i}{2}$. So the number of colors used by this point in the game is at least $i+\frac{(n-1) i}{2}+1$.

Let $m$ be the number of colors necessary for Alice to win the game in this situation, if still possible at all. Let us first consider the case that $r=i+1$. So Bob has just colored in the final partite set (thus giving Alice victory). If $n=3$, then

$$
\begin{aligned}
m & \geq i+\frac{(n-1) i}{2}+1 \\
& =2 r-1 .
\end{aligned}
$$

We note, however, in the case that $n=3$, in light of Lemma 1 , that Alice and Bob are playing with $k \leq 2 r-2$ colors. Thus, the only way that the game has reached this point is if Alice has not played optimally. Hence, we may conclude that if Bob plays with the above strategy, he will win when $n=3$ and $k \leq 2 r-3$. If $n \geq 5$, we have that

$$
\begin{aligned}
m & \geq i+\frac{(n-1) i}{2}+1 \\
& \geq 3 r-2 \\
& \geq 2 r-1 .
\end{aligned}
$$

Thus, in light of Lemma 1, we may again conclude that if Bob plays with the above strategy, he will win when $n \geq 5$ and $k \leq 2 r-2$.

So assume that $r \geq 3, r>i+1$, and there are $j$ remaining partite sets with no colored vertices for some $j \geq 1$. So $r=i+j+1$. As $P_{i+1}$ was the last partite set that Bob was forced to color in first, for the remainder of the game Alice will color in each of the remaining partite sets first, and will use at least $j$ new colors. Bob, using his strategy, will use at least $j-1$ new colors distinct from these. Thus we have 
that

$$
\begin{aligned}
m & \geq i+\frac{(n-1) i}{2}+1+j+(j-1) \\
& =i+2 j+\frac{(n-1) i}{2} .
\end{aligned}
$$

If $n=3$ we have that $m \geq 2 i+2 j=2 r-2$. If $n=5$ we have that $m \geq 3 i+2 j$. Since $i \geq 1$, this gives that $m \geq 2 r-1$, as desired. Note that if $n \geq 7$, we have that $m \geq 4 i+3 j$. Using that $i \geq 1$ this gives that $m \geq 2 r$. With Lemma 1 , using $k \leq 2 r-1$, we have that Alice cannot win, as she has already played suboptimally.

Trivially, note that if $n=1$, then $G=K_{r * n}=K_{r}$ and $\chi_{\mathrm{g}}(G)=r$. If $r=1$, then $G$ is an independent set and $\chi_{\mathrm{g}}(G)=1=2 r-1$. And, as remarked above, if $r=2$, then $G=K_{r * n}=K_{n, n}$ and $\chi_{\mathrm{g}}(G)=3=$ $2 r-1$. Combining this with Lemmas 1 and 2 , we have proven the following theorem:

Theorem 1. Let $r$ and $n$ be positive integers. If $G=K_{r * n}$, then

$$
\chi_{\mathrm{g}}(G)= \begin{cases}r & \text { if } n=1 ; \\ 2 r-2 & \text { if } n=3 \text { and } r \geq 3 ; \\ 2 r-1 & \text { otherwise. }\end{cases}
$$

Therefore, $\chi_{\mathrm{g}}\left(K_{r * n}\right)$ is always bounded above by a function of $r$ and is, for all intents and purposes, independent of $n$.

\section{The 1-Relaxed Game Chromatic Number}

Now we will consider the relaxed coloring game with $K_{r * n}$. We show that for $G=K_{r * n}$, we have that $\chi_{\mathrm{g}}^{1}(G) \rightarrow \infty$ as $n \rightarrow \infty$.

Theorem 2. Let $r$ and $n$ be positive integers with $r \geq 2$. If $G=K_{r * n}$, then $\chi_{\mathrm{g}}^{1}(G)=\left\lceil\frac{n r}{2}\right\rceil$.

Proof. We begin by noting that $\chi_{\mathrm{g}}^{1}(G) \leq\left\lceil\frac{n r}{2}\right\rceil$, since every color can be used at least twice. We suppose that Alice and Bob are playing the $(k-1,1)$-relaxed coloring game on $G$ with $k=\left\lceil\frac{n r}{2}\right\rceil$. Suppose that on her turn Alice colors a vertex in $P_{i}$ with $\alpha$. Bob's strategy will be as follows. He will use color $\alpha$, and he will color a vertex in a partite set different from $P_{i}$ which has the largest number of uncolored vertices. Note that if Bob is successful with this, then Alice will be forced to use a new color on each of her turns. The only possible obstacle for Bob would be if there comes a time in the game when Alice has just colored 
in partite set $P_{i}$ and the only remaining uncolored vertices are in $P_{i}$. We will show that this can never happen.

At the end of turn $t$, let $\varepsilon(t)$ be the difference in the number of uncolored vertices between a partite set with the largest number of uncolored vertices and one with the next largest number of uncolored vertices. Note that $\varepsilon(t)$ is well-defined since $r \geq 2$. We claim that by following his strategy, at the end of each of Bob's turns, $\varepsilon(t) \leq 1$.

We proceed inductively. Clearly, at the end of Bob's first turn, $\varepsilon(2) \leq$ 1. Suppose that on turn $t-1$, Alice colors a vertex in $P_{i}$ with $\alpha$. Since Bob ensured that $\varepsilon(t-2) \leq 1$, then at the end of Alice's turn, $\varepsilon(t-1) \leq 2$, as she can affect the difference by at most 1 . If $\varepsilon(t-1)=0$, then there are at least two partite sets with a maximum number of uncolored vertices. Bob will choose one of them, say $P_{k}$, such that $i \neq k$, and color a vertex in $P_{k}$ with $\alpha$ (in case there is still an uncolored vertex). Thus, at the end of his turn, he has maintained that $\varepsilon(t) \leq 1$.

If $\varepsilon(t-1)=1$ or $\varepsilon(t-1)=2$, then there must be a unique partite set $P_{k}$ with a maximum number of uncolored vertices (again with $i \neq k$ ). Bob will then color a vertex in $P_{k}$ with $\alpha$, resulting in $\varepsilon(t)=\varepsilon(t-1)-1$. Thus, after Bob's turn he has maintained that $\varepsilon(t) \leq 1$. So there will never come a time in the game when, at the beginning of Bob's turn, there are at least two uncolored vertices in a partite set while all other partite sets are completely colored. Hence, Alice is forced to use a new color on each turn. This implies that if only $k-1$ colors are available, then Bob will win, as Alice will not be able to color on her last turn.

\section{Conclusion}

Our direction moving from the example of $K_{n, n}$ was to consider graphs with higher game chromatic number. However, another interesting question that remains open is the following.

Question 2. Is it true that for every nonnegative integer d, there exists a graph $G$ such that $\chi_{\mathrm{g}}^{d}(G)<\chi_{\mathrm{g}}^{d+1}(G)$ ?

While it is clearly true for $d=0$, it is unknown whether this is true for arbitrary $d$.

\section{REFERENCES}

[1] T. Bartnicki, J. Grytczuk, H.A. Kierstead, X. Zhu, "The map-coloring game," Amer. Math. Monthly 114 (2007), no. 9, 783-803.

[2] H. Bodlaender, "On the complexity of some coloring games," Graph Theoretical Concepts in Computer Science," (R. Möhring, ed.), vol. 484, Lecture notes in Computer Science, Springer-Verlag, 1991, 30-40. 
[3] C. Chou, W. Wang, and X. Zhu, "Relaxed game chromatic number of graphs," Discrete Math. 262 (2003), no. 1-3, 89-98.

[4] T. Dinski and X. Zhu, "A bound for the game chromatic number of graphs," Discrete Math. 196 (1999), 109-115.

[5] C. Dunn, H.A. Kierstead, "A simple competitive graph coloring algorithm II," Journal of Combinatorial Theory, Series B, 90 (2004) 93-106.

[6] C. Dunn, H.A. Kierstead, "A simple competitive graph coloring algorithm III," Journal of Combinatorial Theory, Series B, 92 (2004) 137-150.

[7] C. Dunn, H.A. Kierstead, "The relaxed game chromatic number of outerplanar graphs," Journal of Graph Theory, 46 (2004) 69-78.

[8] U. Faigle, W. Kern, H. Kierstead, and W. Trotter, "On the game chromatic number of some classes of graphs," Ars Combinatoria 35 (1993), 143-150.

[9] W. He, J. Wu, and X. Zhu, "Relaxed game chromatic number of trees and outerplanar graphs," Discrete Math. 281 (2004), no. 1-3, 209-219.

[10] H. Kierstead, "A simple competitive graph coloring algorithm," Journal of Combinatorial Theory, Series B, 78 (2000), 57-68.

Department of Mathematics, Linfield College, McMinnville, OR 97128

E-mail address: cdunn@linfield.edu

URL: http://catfiles.linfield.edu/People/Faculty/cdunn/public 